Surgery Journal 6 (1): 13-17, 2011

ISSN: $1816-3211$

(C) Medwell Journals, 2010

\title{
Bloodless Removal of a Primary Posterior Wall Oropharyngeal Carcinoma by Transoral Approach with the Ligasure Instrument, Case Report
}

\author{
${ }^{1}$ Zouloumis Lampros, ${ }^{1}$ Tilaveridis Ioannis, ${ }^{1}$ Lazaridis Nicolas and ${ }^{2}$ Koutsonikolas Dimitrios \\ ${ }^{1}$ Department of Oral and Maxillofacial Surgery, \\ ${ }^{2}$ Department of Physiology, Aristotle University of Thessaloniki, Greece
}

\begin{abstract}
Oropharyngeal tumors are usually quamous cells carcinomas and rarely minor salivary tumors, lymphomas or lymphepitheliomas. Cancer of the posterior pharyngeal wall is relatively rare and needs a multidisciplinary approach. The treatment of oropharyngeal carcinoma remains controversial. Surgical treatments, radiotherapy, chemotherapy as well as a combination of these methods have been used. The extraoral approach with mandibular swing and the intraoral approach have been applied for resection of oropharyngeal cancers. The intraoral approach avoids the consequences of mandibular osteotomy and saves surgical time but it necessitate the, absolute control of intraoperative bleeding. In the study, reseachers present the case of a 40 years old woman with an exophytic squamous-cell carcinoma of the posterior pharyngeal wall. The tumor was staged as T2N0 according to clinical and imaging evaluation. The tumor was removed intraorally with the ligasure precise instrument, achieving an entirely bloodless operative field. A lyophilized dura was sutured to the defect of the posterior pharyngeal wall. Histopatologic evaluation showed verrucous carcinoma and the specimen was positive for Human Papilloma Virus (HPV 16). The patient received postoperative radiotherapy $5.500 \mathrm{cGy}$ on the primary site and $5.000 \mathrm{cGy}$ on the neck. Bone scanning with Tc- $99 \mathrm{~m}$, performed postoperatively was negative for metastasis. The postoperative course was uneventful and 19 months after surgery, the patient remains free of disease according to clinical and imaging evaluation without any impairment of the pharyngeal function.
\end{abstract}

Key words: Carcinoma, posterior pharyngeal wall, surgery, ligasure, hemostasis, radiotherapy

\section{INTRODUCTION}

The oropharynx is characterized by a rich blood and lymphatic system. The anterior wall includes the base of the tongue, the superior wall includes the soft palate and the lateral wall includes both anterior and posterior pillars and palatine tonsil. The upper border of the oropharynx is defined by a line passing from the soft palate to the posterior pharyngeal wall and the inferior border is defined by a horizontal plane at the level of the vallecula (Lim et al., 2008; Shimizu et al., 2006). The oropharyngeal squamous-cell carcinoma represents a small percentage of the tumors of the upper aero-digestive tract, accounting for $1-3 \%$ in different countries and its tendency has increased in the last decades (Greenlee et al., 2001; Lim at al., 2008). Smoking and excessive alcohol consumption along with HPV are well-known risk factors (Hermans, 2005; Walvekar et al., 2008). The increase in the incidence of oropharyngeal carcinoma is more intense in young patients and is attributed to HPV infection (Sturgis and Cinciripini, 2007). Isolated cancers of the posterior oropharyngeal wall are rare and this wall is usually invaded by neighboring cancer originating from the lateral pharyngeal wall. Retrospective studies on oropharyngeal cancers from different countries, reveal that true squamous-cell carcinoma of the posterior pharyngeal wall are a really rare entity (Shimizu et al., 2006; Lim et al., 2008; Fco et al., 2009). CT and MRI are helpful tools in the clinical staging of the lesion (Mancuso et al., 1983; Okumura et al., 1998). Surgery, radiotherapy or chemotherapy, sole or in combination are possible treatment modalities which are used for treatment of SSC of the posterior pharyngeal wall. Fixation due to invasion of the prevertebral fascia upstaging the cancer precludes, the possibility of surgical removal and is related with the worst prognosis (Hermans, 2005). In the present study, reseachers present a case of a primary exophytic squamous-cell carcinoma of the posterior pharyngeal wall which was successfully treated with transoral surgical approach with the aid of the Ligasure vessel sealing system which is a new surgical instrument assuring the absolute control of intraoperative bleeding. Complementary, chemoradiation was also aplied. Reseachers also study the key points of the intraoral 3 approach for this location of tumors and the controversy surrounding their treatment.

Corresponding Author: Lampros Zouloumis, 17 Ippodromiou, P.C. 54-248, Thessaloniki, Greece 


\section{MATERIALS AND METHODS}

Case report: The patient was a 40 years old female referred by her primary care physician for diagnosis and treatment of an exophytic mass of the oropharynx. Before her admission, the patient had some difficulty in deglutition without any other symptoms. Physical examination revealed a healthy woman without other medical problems.

Social history revealed that she smoked one and a half pack of cigarettes per day for the last 10 years and she consumed a moderate amount of alcohol. Oral examination revealed an exophytic mass sitting on the posterior wall of the oropharynx (Fig. 1). Clinical evaluation of the remaining part of the oropharynx and the oral cavity was negative. Flexible fiberoptic examination of the upper aerodigestive tract was unremarkable and

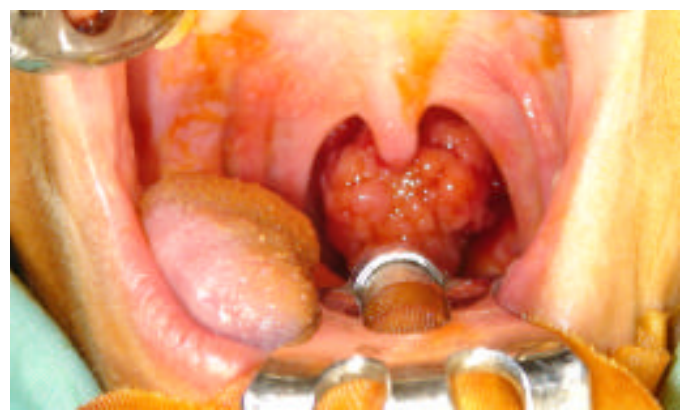

Fig. 1: Primary tumor of the posterior pharyngeal wall
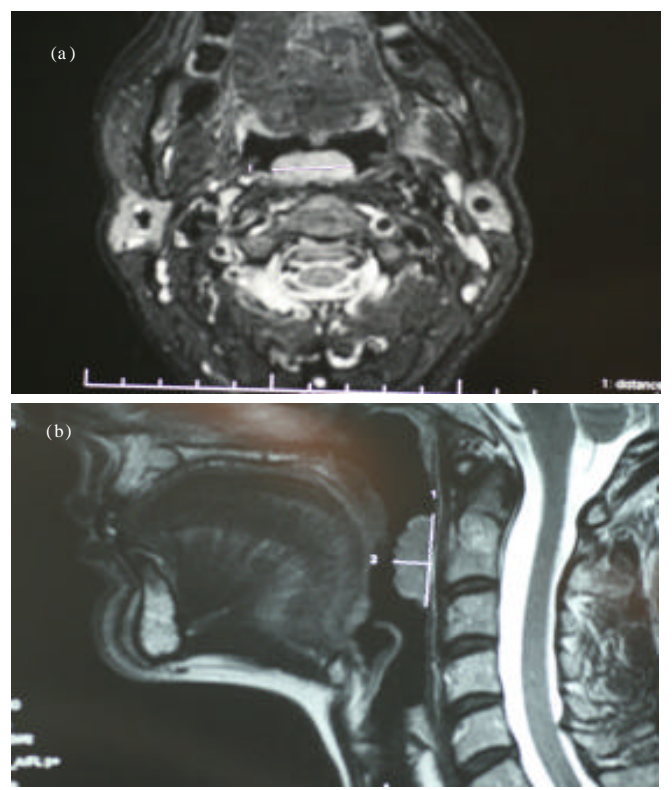

Fig. 2: a) Transverse section of MRI showing the dimension of the lesion and b) The appearance of tumor in the sagital view tests were normal. CT scan and MRI revealed a large exophytic mass located at the posterior wall of the oropharynx with its greater dimensions ranging palpation of the neck was negative. Preoperative chest X-ray, cell blood count, electrolytes and liver function $22 \times 27 \times 10 \mathrm{~mm}$ (Fig. 2a, b). There was absence of enlarged lymph nodes along the internal jugular veins or in the retropharyngeal space. Biopsy under local anaesthesia revealed a squamous-cell carcinoma of the pharyngeal wall and the patient was classified as T2N0 stage. The patient was operated under general anaesthesia in order to perform an intraoral excision of the tumor. A Dingman retractor was used to achieve the maximal opening of the mouth to have better access to the posterior wall of the oropharynx. The borders of the tumor were demarcated and the lesion was removed totally with the use of Ligasure thermal system. The excision included the

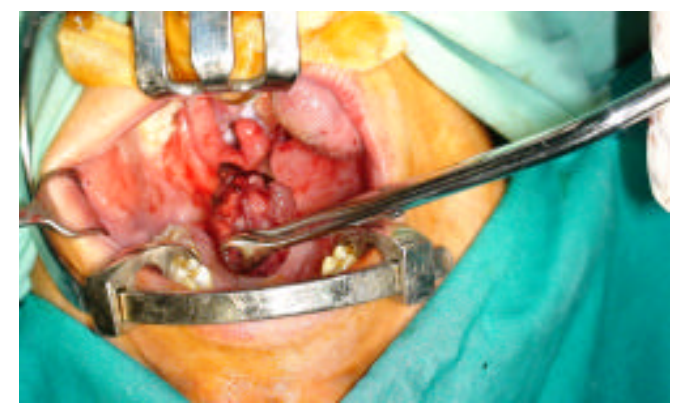

Fig. 3: Excision of the lesion from the posterior pharyngeal wall

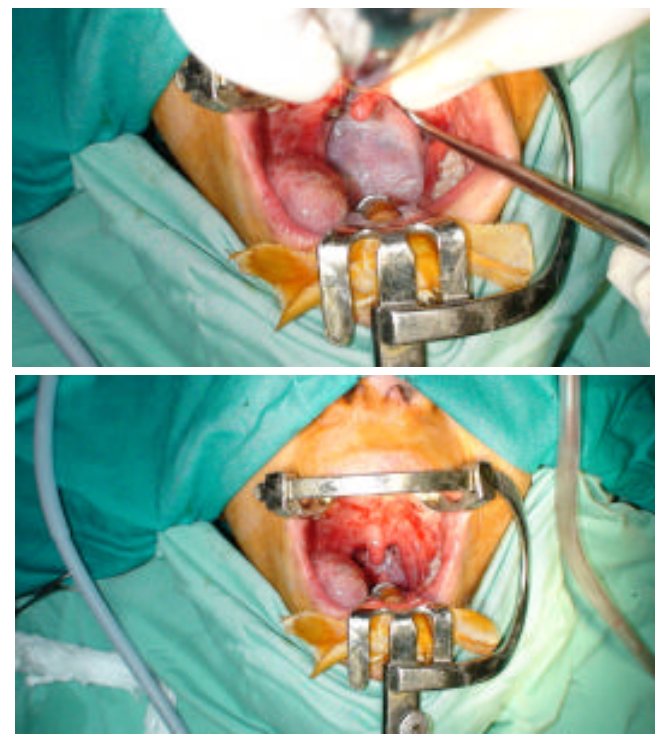

Fig. 4: a) A resorbable membrane has been used to cover the defect and $b$ ) The surgical field after membrane placement 


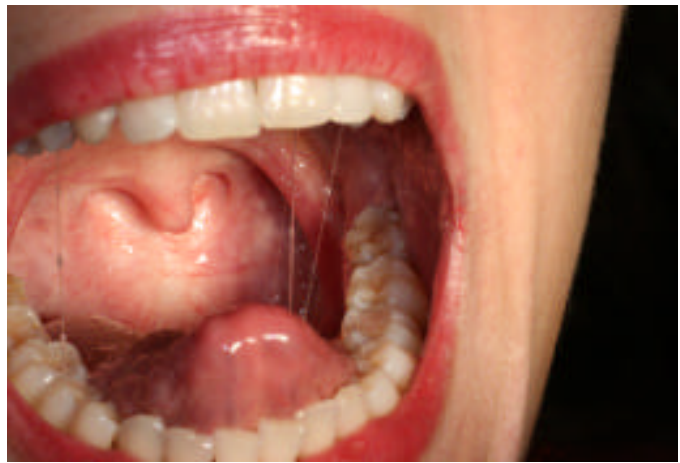

Fig. 5: Postoperative view, 19 months after the operation
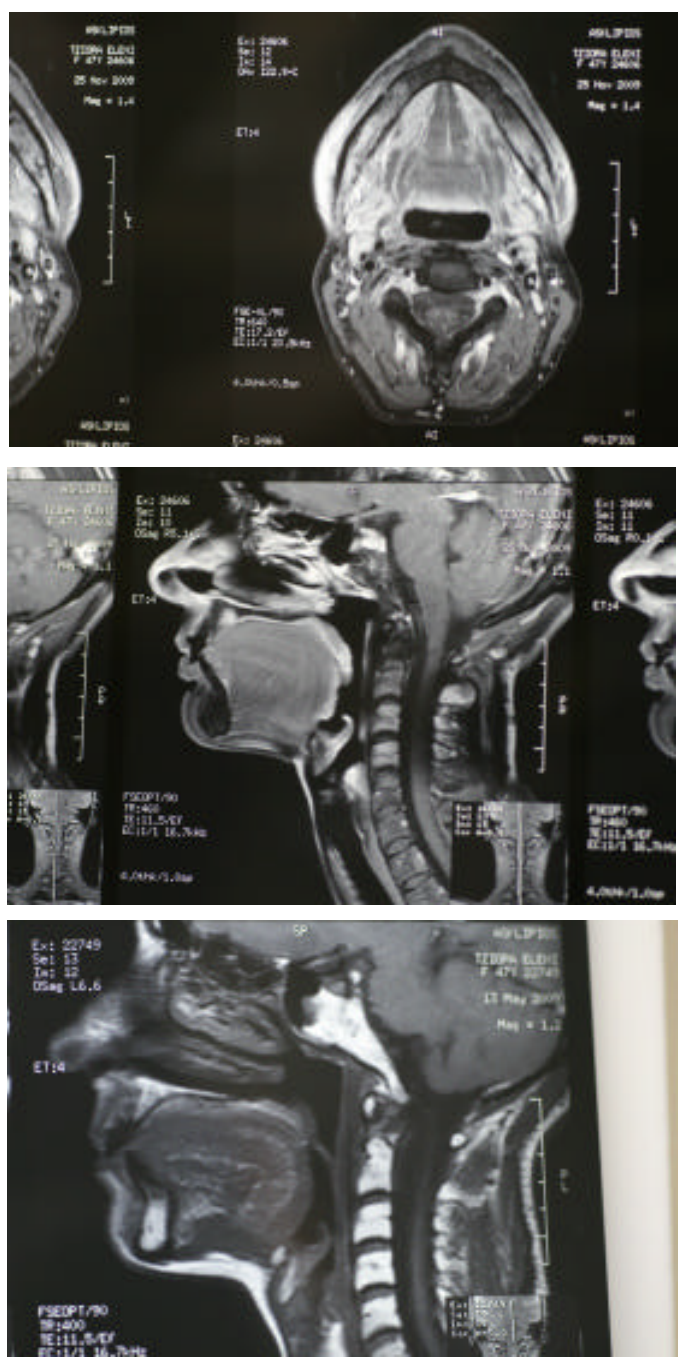

Fig. 6: a) Axial view of patient's MRI showing complete healing of the primary tumor and absence of recurrence; b) Sagital view shows the same findings and c) MRI shows the normal swallowing function prevertebral fascia as according to the preoperative imaging, there was no infiltration of the fascia. The defect of the posterior pharyngeal wall was covered with duraper and sutured at its periphery with absorbable sutures (Fig. 3 and $4 a, b$ ).

The postoperative period was uneventful and the patient recovered without any complication. The pathologic examination confirmed the initial diagnosis and further examination of the specimen revealed an intense positive test for the HPV16 virus. The patient underwent evaluation by the medical oncology team and she received adjunctive radiation therapy. About $5.500 \mathrm{cGy}$ were administered on the primary site and $5.000 \mathrm{cGy}$ on the neck. Bone scanning with Tc-99m, performed was negative for metastasis. About 2 years later, the patient was symptom and disease free according to the clinical and imaging examination (Fig. 5 and $6 a-c)$. The functions of speech and deglutition were undisturbed.

\section{RESULTS AND DISCUSSION}

Oropharyngeal carcinoma is a tumor with low incidence, accounting for 0.3-0.5 of all malignancies and is a multidisciplinary problem (Johansen et al., 1990). Peak incidence has been observed in the 6th and 7th decades of life and there is male predominance. Very interesting trends according to statistics are that the patient population is becoming younger than in the past. Tobacco and alcohol consumption are the most important risk causing changes to all mucosa of the head and neck, prolonging exposure to mitogens leading to field cancerization.

Human papilloma virus infectious disease and some of its types, especially virus 16 are most commonly related with head and neck cancers. This is true in the case which was intensely positive for HPV16. Isolated squamous-cell carcinoma of the posterior wall of the oropharynx is rare. In most cases, these cancers develop after infiltration by tumors originating from neighbouring tissues and especially from the lateral pharyngeal wall (Hermans, 2005).

$\mathrm{CT}$ and MRI imaging is of utmost importance in order to delineate the extent and to define the staging of the cancer, demonstrating the involvement of the retropharyngeal fat (Hsu et al., 2005). However, imaging signs such as obliteration of the retropharyngeal space, enlargement and asymmetry of the pre-vertebral muscles and thickening abnormalities are unreliable to define the tumor extension into the pre-vertebral space (Loevner et al., 1998; Hsu et al., 2005). The treatment of this oropharyngeal cancer is a multidisciplinary team approach. This team includes a reconstructive surgeon, 
an oncologist, a radiotherapist and a speech pathologist. Each one plays an individual key role in treatment planning. In cancer patients, all efforts are focused on the preservation of the organ and its functions. This is also true for oropharyngeal cancers as the functions of this anatomic region are intimately related to deglutition, speech and swallowing (O'Connell et al., 2008).

The optimal treatment modality for oropharyngeal cancer is disputed but it is accepted that in early stages, surgery and radiotherapy yield similar results. In advanced cancer, surgery with postoperative radiotherapy is a good treatment option (Lim et al., 2008). Radiotherapy for treatment of cancers in this location has many immediate as well as long term complications such as xerostomia, dysphagia, trismus and osteoradionecrosis of the mandible but avoids the consequences of surgical operation (Lee et al., 1993; Spiro et al., 1981).

Neck dissection in neck involvement along with open neck exploration in retropharyngeal adenopathy with immediate evaluation of the prevertebral muscles is another treatment option, even though such lesions are also treated by radiotherapy or chemoradiation as the cure rates are comparable to those of surgery (Million et al., 1994).

Radiotherapy in this location is controversial because of the vicinity of the spinal cord and also due to the fact that a full dose of radiation therapy could lead to severe mucosal necrosis and laryngeal edema, associated with pain, tracheotomy and tube feeding (Pene et al., 1978).

The transoral access of tumors of the posterior pharyngeal wall is a challenge because of its and the bloodless intraoperative field is of utmost importance for the radicality of the operation. The Dingman retractor that used for the operation in cleft palate, patient is helpful for good visualization of the posterior pharyngeal wall. The 2nd key point for secure removal of the tumor from this location is the bloodless excision. The latter achieved with the Ligasure vessel sealing system. This devise can effectively dissect tissue bands and seal vessels 1-7 mm in diameter with minimal thermal spread to the surrounding tissues. The Ligasure system provides strength of occluded vessel higher than the classic bipolar coagulators and equal to the hemoclip and ligation with sutures (Kennedy et al., 1988; Heniford et al., 2001). Last years, this device has been used not only in general surgery but in wide field of head and neck surgery (Prokopakis et al., 2005; Lepner and Vaasna, 2007; Pons et al., 2009).

For small primary tumors of the posterior pharyngeal wall, it is easy to perform a resection and close the defect directly or suture the pharyngeal mucosa to the prevertebral fascia, sometimes with a split-thickness skin graft. However in large tumors, there is the need to use regional or free vascularized flaps. The most useful flaps for reconstruction of this area are the myocutaneous platysma flap and the forearm free with the latter being more reliable.

The forearm flap can also be used in previously irradiated patients (O'Connell et al., 2008; Walvekar et al., 2008). In the patient, the lesion recorded as $\mathrm{T} 2$ was superficially spreading and there was no need to use any of the aforementioned flaps. Instead, the posterior pharyngeal defect was covered with an absorbable membrane which was sutured to the prevertebral fascia. The lesion was removed intraorally, using the Dingman retractor to achieve the maximum access to the posterior pharyngeal wall.

The excision of the primary tumor was performed with the use of gun type ligasure with absolute control of the intraoperative bleeding.

\section{CONCLUSION}

The combination of ligasure instrument with the use of Dingman's retractor assures the bloodless removal of tumors located at the posterior pharyngeal wall and helps to avoid the extraoral approach and the subsequent creation of a noticeable facial scar. It also minimizes, the postoperative symptoms and the patient quickly return to the normal diet.

\section{REFERENCES}

Fco, P.R., C.L. Molla, F.M.J. Ramirez, E.E. Ferriol, M.T. Conejeros, P.E. Navas and J.D. Galofre, 2009. Prognostic factors in oropharynx carcinoma. J. Chin. Clin. Med., 7: 378-384.

Greenlee, R.T., M.B. Hill-Harmon, T. Murray and M. Thun, 2001. Cancer statistics. Cancer J. Clin., 51: $15-36$.

Heniford, B.T., B.D. Matthews, R.F. Sing, C. Backus, B. Pratt and F.L. Greene, 2001. Initial results with an electrothermal bipolar vessel sealer. Surg. Endoscopy, 15: $799-801$.

Hermans, R., 2005. Multidisciplinary symposium: Head and neck cancer. Cancer Imag., 5: 52-57.

Hsu, W.C., L.A. Loevner, R. Karpati, T. Ahmed and A. Mong et al., 2005. Accuracy of magnetic resonance imaging in predicting absence of fixation of head and neck cancer to the prevertebral space. Head Neck, 27: 95-100. 
Johansen, L.V., J. Overgaard, M. Overgaard, N. Birkler and A. Fisker, 1990. Squamous cell carcinoma of the oropharynx: An analysis of 213 consecutive patients scheduled for primary radiotherapy. Laryngoscpe, 100: 985-990.

Kennedy, J.S., P.L. Stranahan, K.D. Taylor and J.G. Chandler, 1988. High-burst-strength, feedbackcontrolled bipolar vessel sealing. Surg. Endoscopy, 12: 876-878.

Lee, W.R., W.M. Mendenhall, J.T. Parsons, R.R. Million, N.J. Cassisi and S.P. Stringer, 1993. Carcinoma of the tonsillar region: A multivariate analysis of 243 patients treated with radical radiotherapy. Head Neck, 15: 283-288.

Lepner, U. and T. Vaasna, 2007. Ligasure vessel sealing system versus conventional vessel ligation in thyroidectomy. Scandinavian J. Surg., 96: 31-34.

Lim, Y.C., H.J. Hong, S.J. Baek, J.H. Park and G.E. Kim et al., 2008. Combined surgery and postoperative radiotherapy for oropharyngeal squamous cell carcinoma in Korea: Analysis of 110 cases. Int. J. Oral Maxillofacial Surg., 37: 1099-1105.

Loevner, L.A., I.L. Ott, D.M. Yousem, K.T. Montone and E.R. Thaler et al., 1998. Neoplastic fixation to the prevertebral compartment by squamous cell carcinoma of the head and neck. Am. J. Roentgenol., 170: 1389-1394.

Mancuso, A.A., H.R. Harnsberger, A.S. Muraki and M.H. Stevens, 1983. Computed tomography of cervical and retropharyngeal lymph nodes: Normal anatomy, variants of normal and applications in staging head and neck cancer. Part II: pathology. Radiology, 148: 715-723.

Million, R.R., N.J. Cassini and A.A. Mancuso, 1994. Hypopharynx: Pharyngeal Walls, Pyriform Sinus, Postcricoid Pharynx. In: Management of Head and Neck Cancer: A Multidisciplinary Approach, Million, R.R., N.J. Cassini (Eds.). J.B. Lippincott and Co., Philadelphia, pp: 516.
Okumura, K., Y. Fujimoto, Y. Hasegawa, H. Matsuura and B. Nakayama et al., 1998. Retropharyngeal node metastasis in cancer of the oropharynx and hypopharynx: analysis of retropharyngeal node dissection regarding preoperative radiographic diagnosis. Nippon Jibiinkoka Gakkai Kaiho., 101: 573-577.

O'Connell, D.A., J. Rieger, J.R. Harris, P. Dziegielewski and J. Zalmanowitz et al., 2008. Swallowing function in patients with base of tongue cancers treated with primary surgery and reconstructed with a modified radial forearm free flap. Arch. Otolaryngol. Head Neck Surg., 134: 857-864.

Pene, F., V. Avedian, F. Eschwege, A. Barrett, G. Schwaab, P. Marandas and C. Vandenbrouck, 1978. A retrospective study of 131 cases of carcinoma of the posterior pharyngeal wall. Cancer, 42: 2490-2493.

Pons, Y., J. Gauthier, E. Ukkola-Pons, P. Clement, E. Roguet, J.L. Poncet and C. Conessa, 2009. Comparison of LigaSure vessel sealing system, harmonic scalpel and conventional hemostasis in total thyroidectomy. Otolaryngol. Head Neck Surg., 141: 496-501.

Prokopakis, E.P., V.A. Lachanas, E.S. Helidonis and G.A. Velegrakis, 2005. The use of the Ligasure Vessel Sealing System in parotid gland surgery. Otolaryngol. Head Neck Surg., 133: 725-728.

Shimizu, K., H. Inoue, M. Saitoh, N. Ohtsuki and H. Ishida et al., 2006. Distribution and impact of lymph node metastases in oropharyngeal cancer. Acta Otolaryngol., 126: 872-877.

Spiro, R.H., F.P. Gerold and E.W. Strong, 1981. Mandibular swing approach for oral and oropharyngeal tumors. Head Neck Surg., 3: 371-378.

Sturgis, E.M. and P.M. Cinciripini, 2007. Trends in head and neck cancer incidence in relation to smoking prevalence: An emerging epidemic of human papillomavirus-associated cancers? Cancer, 110: 1429-1435.

Walvekar, R.R., R.J. Li, W.E. Gooding, M.K. Gibson, D. Heron, J.T. Johnson and R.L. Ferris, 2008. Role of Surgery in Limited (T1-2, N0-1) Cancers of the Oropharynx. Larynscope, 118: 2129-2134. 